\title{
Raman Cell Optimisation for Distributed Amplification Based Transmission Systems
}

\author{
Giuseppe Rizzelli ${ }^{1}$, Francesca Gallazzi ${ }^{1}$, Pawel Rosa ${ }^{2}$, Pedro Corredera Guillén ${ }^{1}$, \\ and Juan Diego Ania-Castañón ${ }^{1}$ \\ ${ }^{1}$ Instituto de Óptica "Daza de Valdés", CSIC, 28006 Madrid, Spain \\ ${ }^{2}$ National Institute of Telecommunications, Warsaw, Poland \\ Tel: (0034) 915616800, Fax: (0034)915645557, e-mail: giuseppe.rizzelli@,csic.es
}

\begin{abstract}
Commercially available Raman fiber pumps display relatively high Relative Intensity Noise (RIN) that gets transferred to the output signal in systems relying on Raman distributed amplification. This has been shown to drastically hinder performance in Raman-based transmission systems [1], limiting the practically usable forward pump powers and setting constraints to the design of low signal power variation (SPV) bidirectional systems with optimised optical signal-to-noise ratios (OSNR) [2]. Recent works have highlighted a remarkable improvement in the resilience to RIN transfer of higher-order ultralong Raman fiber lasers (URFL) in a random Distributed Feedback (rDFB) laser amplifiers configurations with no fiber Bragg grating (FBG) at the front-end [3]. Moreover, we have shown [4] that a low-reflectivity front-end FBG can be more beneficial than no backreflections at all, for enhancing pumping efficiency while still keeping RIN transfer under control.

Here we extend previous analysis of the low-reflectivity URFL amplification scheme including experimental investigation of RIN transfer, OSNR and pump power requirements for different architectures. We perform a comparison with the rDFB approach and present a detailed optimisation of the main amplifier parameters for the effective design of Raman-based transmission systems.
\end{abstract}

Keywords: Raman, relative intensity noise, FBG, reflectivity, random distributed feedback amplifier, distributed amplification.

\section{INTRODUCTION}

Ultralong Raman fiber laser (URFL) [1,2] architectures are being increasingly deployed to overcome EDFA limitations in terms of transmission bandwidth, distance and noise performance, yielding remarkable results in discrete-based as well as unrepeatered and long-haul transmission scenarios [4-7]. In order to take full advantage of the benefits provided by distributed amplification over traditional EDFA amplifiers, a careful analysis of the different noise contributions needs to be carried out prior to determining which Raman cell configuration is the most suitable for a specific application. Two main noise sources adversely affect the performance of a Raman amplifier: Amplified Spontaneous Emission (ASE) noise and Relative Intensity Noise (RIN). ASE noise can be easily minimized through co-pumping, i.e. by increasing the amount of pump power co-propagating with the signal in the forward $(\mathrm{FW})$ direction, thus preventing the signal from experiencing excessive attenuation before being amplified. This improves the noise figure of the amplifier and, therefore, the received Optical Signal-toNoise Ratio (OSNR) of the system at the cost of increased nonlinear effects. On the other hand the RIN transferred from the pumps to the signal is mainly delivered by the FW propagating pump, which makes it a less trivial issue to deal with [8-12]: the simple removal of the FW pump would drastically reduce the OSNR. Novel, RIN-tolerant distributed amplification schemes such as the random Distributed Feedback (rDFB) laser [3] have recently attracted much attention as they exhibit signal RIN levels comparable to the purely counter-pumping configuration even for a FW pump contribution close to $50 \%$ of the total pump power [13]. The symmetrical pumping ideally allows to obtain an optimal trade-off between OSNR and generated nonlinearities at the price of a reduced pumping efficiency.

In this paper we present a detailed optimization of the cavity parameters of a $2^{\text {nd }}$-order URFL performed through the monitoring of key performance indicators such as signal output RIN, optical signal-to-noise ratio (OSNR) and pump power requirement when length, FW pump ratio (FPR) and front-end FBG reflectivity are varied.

\section{EXPERIMENTAL LAYOUT}

The experimental setup presented in Fig. 1 includes a pair of FBGs at $1455 \mathrm{~nm}$ located at the front and end of the span to reflect stokes shifted light from the $1366 \mathrm{~nm}$ pumps. The reflectivity of the front FBG in the $2^{\text {nd }}$ order URFL configuration was supplied by a variable reflectivity module (VRM) that consists of a high reflectivity FBG and a variable optical attenuator (VOA). The reflectivity of the FBG at the receiver side is fixed at $\sim 95 \%$. To monitor the backscattered light and the actual power inside the cavity we installed two 99/1 couplers. The transmission medium is standard SMF. In order to keep the pumps RIN level unaltered, the forward and backward pump power is fixed and the launch pump power into the fibre was varied using a VOA. The total pump power needed to achieve zero net gain, the OSNR over a $0.1 \mathrm{~nm}$ bandwidth, and the output signal RIN 
have been measured as a function of the forward pump ratio to total pump power (FPR) for cavity lengths and front-end reflectivities ranging from $10 \mathrm{~km}$ to $150 \mathrm{~km}$ and from 0 to $20 \%$ respectively. Reflectivity was calculated as the ratio between the optical powers at Port 2 and Port 1 measured through an optical signal analyzer at the FBG central wavelength, whereas the FPR is the ratio of the FW pump power to the total pump power required to have full recovery of the span loss at the amplifier output.

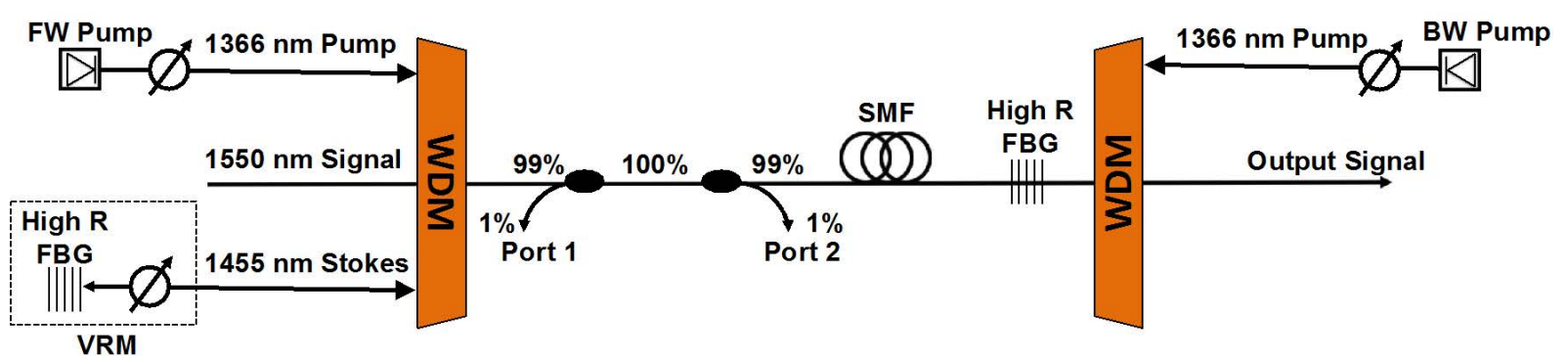

Figure 1. Experimental setup of a $2^{\text {nd }}$-order Raman amplifier with variable front-end reflectivity.

\section{RESULTS AND DISCUSSION}

Figure 2 shows how the total pump power evolves due to an increase of the VRM reflectivity as we gradually change the pumping scheme from purely counter-propagating (0 FPR) to co-propagating (1 FPR). For the sake of clarity, we only show in Fig. 2a a comparison between a half-open cavity with $0 \%$ reflectivity at the front-end (rDFB) and a partially closed cavity with $5 \%$ front reflectivity.

Figure 2a highlights the beneficial effect of having a front reflector: a reflectivity as low as $5 \%$ is enough to reduce the necessary pump power by $2 \mathrm{~dB}$ and $1.5 \mathrm{~dB}$ for a $25 \mathrm{~km}$ and a $50 \mathrm{~km}$ cell respectively. In the longer cells the pumps experience a higher attenuation, hence the backward (BW) pump power reaching the input side is too low to have any meaningful effect on FW Stokes generation and the 5\% reflectivity cavity behaves similarly to the rDFB in configurations with low FW pumping. However, as the FW pump contribution grows, the FW $1455 \mathrm{~nm}$ Stokes is more efficiently generated thanks to the feedback provided by the front FBG and the overall pumping efficiency improves. The $10 \mathrm{~km}$ cavity case depicted in Fig. $2 \mathrm{~b}$ shows an overall $6 \mathrm{~dB}$ power efficiency improvement at a 50\% FPR using a 20\% FBG rather than a rDFB architecture.
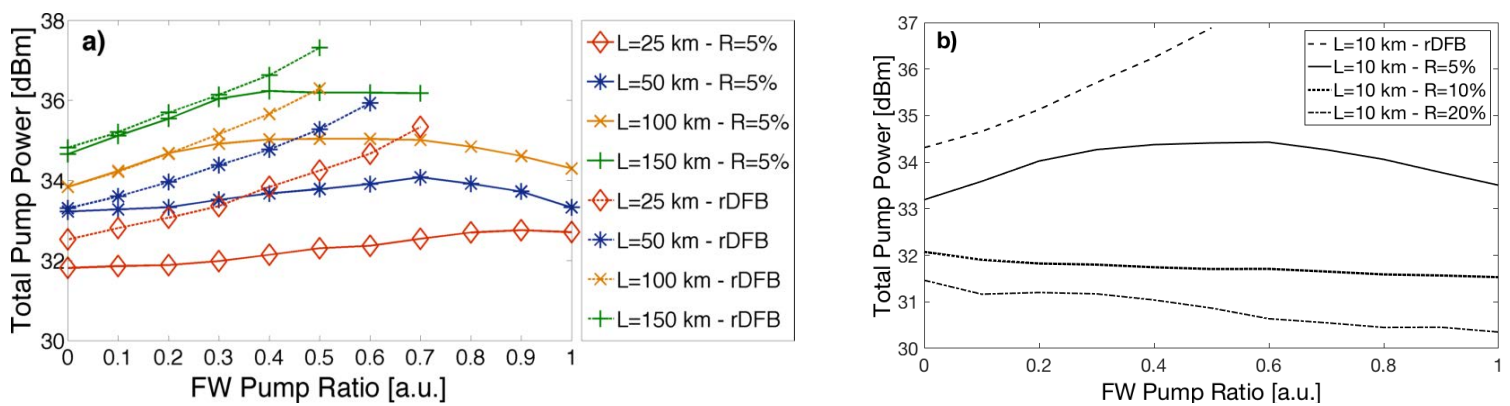

Figure 2. Total pump power as a function of the FPR for: (a) four cavity lengths in the rDFB and 5\% reflectivity cases and (b) for a $10 \mathrm{~km}$ cavity and reflectivity from $0 \%$ to $20 \%$.

Figure 3 shows the output signal RIN normalized to the specific cavity length, integrated over a [9 kHz, $1 \mathrm{MHz}] \mathrm{RF}$ frequency interval for the five investigated cavity lengths, as a function of the FW pump ratio. The rDFB scheme with no front-FBG is clearly the best performing as the RIN stays below $-120 \mathrm{~dB} / \mathrm{km}$ for all the measurable FPRs except in the $10 \mathrm{~km}$ cavity, where the required pump power is substantially higher than in most of the other designs. As the front reflectivity increases, longer cavities produce less RIN-per-km for FPR up to $30 \%$. Beyond this FPR value, a longer interaction length between the pump and the signal causes the RIN to build up in a gradually stronger fashion.

The normalized OSNR measured at the amplifiers output over a $0.1 \mathrm{~nm}$ bandwidth as a function of the FPR is presented in Fig. 4. Please note that both the OSNR and the RIN in Fig. 3 have been normalized to the cavity length, in order to have a better estimation of the best-performing configuration independently of the required number of spans. The maximum OSNR-per-km decreases for longer cavity designs due to a larger power variation experienced by the signal along the fiber, which results in higher ASE generated in the amplification process. As the FW pump contribution increases the output OSNR grows as expected. Interestingly, this is not the case for short cavities in rDFB configuration: the two shortest amplifiers are the ones that perform best with pure BW pumping, as the SMF length is short enough for the BW pump to amplify the FW-propagating Stokes at the beginning of the span, providing similar benefits to those found in the cases in which a FW pump is present. 
$\vartheta \mathrm{L}=10 \mathrm{~km} \vartheta \mathrm{L}=25 \mathrm{~km} * \mathrm{~L}=50 \mathrm{~km} * \mathrm{~L}=100 \mathrm{~km}+\mathrm{L}=150 \mathrm{~km}$
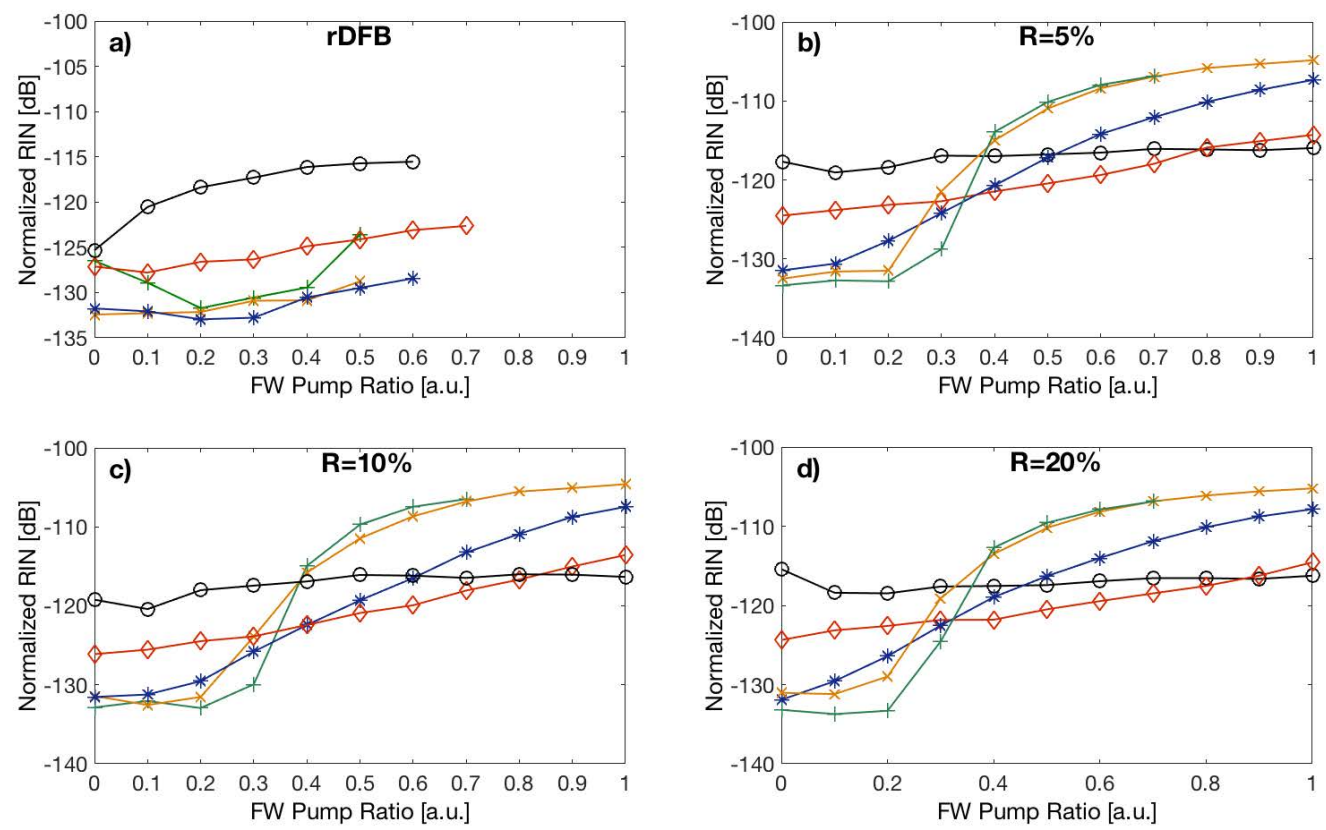

Figure 3. Output normalized signal RIN integrated over the [9 $\mathrm{kHz}, 1 \mathrm{MHz}] \mathrm{RF}$ frequency range for different cavity lengths in the: (a) rDFB, (b) $5 \%$, (c) 10\%, and (d) $20 \%$ reflectivity cases.
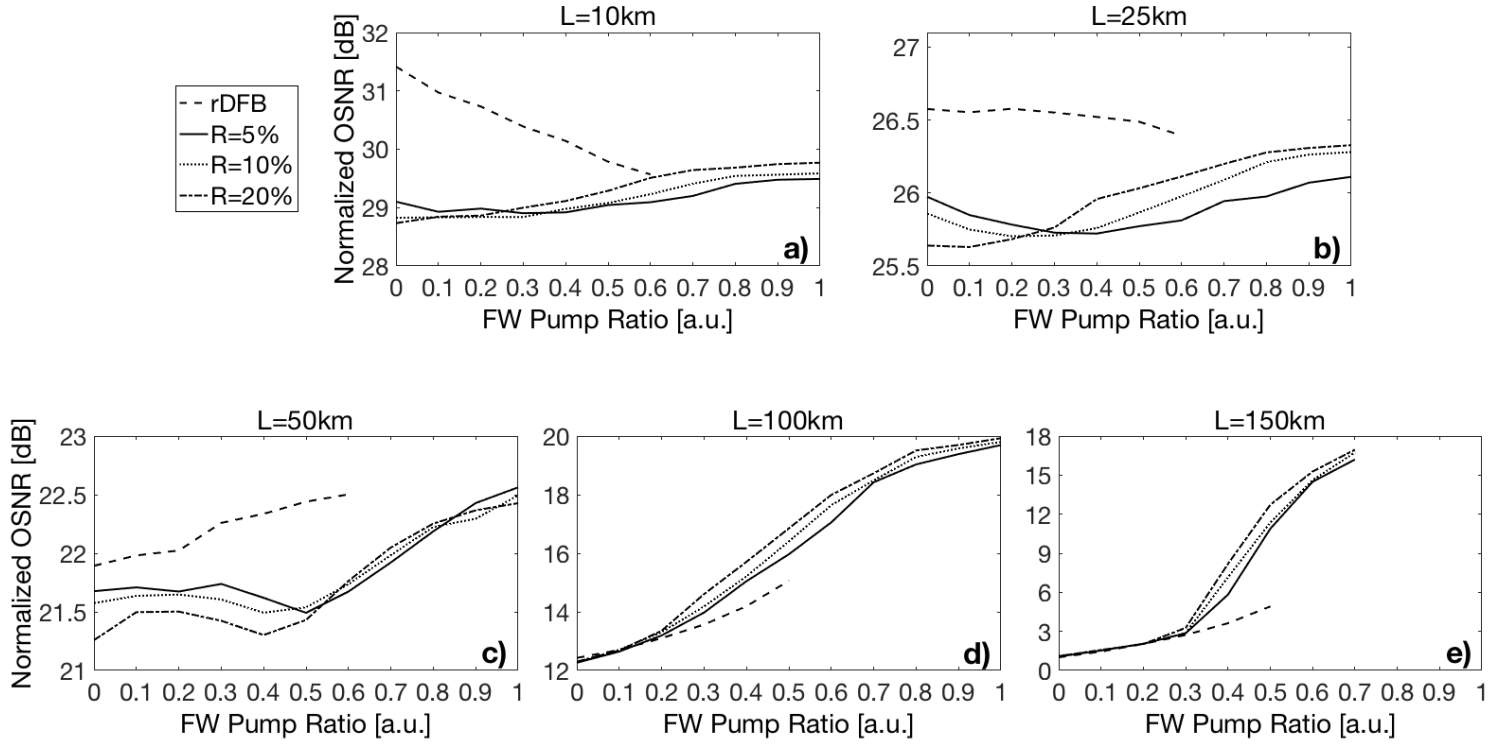

Figure 4. Output normalized OSNR for different front-end reflectivities in the: (a) $10 \mathrm{~km}$, (b) $25 \mathrm{~km}$, (c) $50 \mathrm{~km}$, (d) $100 \mathrm{~km}$, and (e) $150 \mathrm{~km}$ cavities.

\section{CONCLUSION}

We have presented an experimental validation of the performance of a $2^{\text {nd }}$-order Raman amplifier for application to the efficient design of transmission systems based on distributed amplification. We found that rDFB architectures are to be preferred when the cavity length can be chosen to be shorter than $100 \mathrm{~km}$ provided that the additional required pump power is taken into account.

\section{ACKNOWLEDGEMENTS}

This work has been sponsored by FP7 ITN programme ICONE (608099); MSCA-IF 'CHAOS' (658982) for P. Rosa; Spanish MINECO grant ANOMALOS (TEC2015-71127-C2); Comunidad de Madrid grant SINFOTON (S2013/MIT-2790-SINFOTON-CM). 


\section{REFERENCES}

[1] J. D. Ania-Castañón: Quasi-lossless transmission using second-order Raman amplification and FBG, Opt. Express, vol. 12, pp. 4372-4377, 2004.

[2] J. D. Ania-Castañón, T. J. Ellingham, R. Ibbotson, X. Chen, L. Zhang, and S. K. Turitsyn: Ultralong Raman fiber lasers as virtually lossless optical media, Phys. Rev. Lett., vol. 96, p. 023902, 2006.

[3] S. K. Turitsyn, S. A. Babin, A. E. El-Taher, P. Harper, D. V. Churkin, S. I. Kablukov, J. D. Ania-Castañón, V. Karalekas, and E. V. Podivilov: Random distributed feedback fibre laser, Nature Photonics, vol. 4, pp. 231-235, 2010.

[4] P. Rosa, J. D. Ania-Castañón, and P. Harper: Unrepeatered DPSK transmission over 360 km SMF-28 fibre using URFL based amplification, Opt. Express, vol. 22, pp. 9687-9692, 2014.

[5] M. Tan, P. Rosa, S. Thai Le, I. D. Phillips, and P. Harper: Evaluation of 100G DP-QPSK long-haul transmission performance using second order co-pumped Raman laser based amplification, Opt. Express, vol. 23, pp. 22181-22189, 2015.

[6] M. Tan, P. Rosa, S. T. Le, Md. A. Iqbal, I. D. Phillips, and P. Harper: Transmission performance improvement using random DFB laser based Raman amplification and bidirectional second-order pumping, Opt. Express, vol. 24, pp. 2215-2221, 2016.

[7] M. Tan, P. Rosa, I. Phillips, and P. Harper: Extended reach of $116 \mathrm{~Gb} / \mathrm{s}$ DP-QPSK transmission using random DFB fiber laser based Raman amplification and bidirectional second-order pumping, in Proc. OFC 2015, Los Angeles, USA, Mar. 2015, paper W4E.1.

[8] C. R. S. Fludger, V. Handerek, and R. J. Mears: Pump to signal RIN transfer in Raman fiber amplifiers, J. Lightwave Technol., vol. 19, pp. 1140-1148, 2001.

[9] M. Krause, S. Cierullies, H. Renner, and E. Brinkmeyer: Pump-to-Stokes RIN transfer in Raman fiber lasers and its impact on the performance of co-pumped Raman amplifiers, Opt. Commun., vol. 260, pp. 656-661, 2006.

[10] M. Alcón-Camas, and J. D. Ania-Castañón: RIN transfer in 2nd-order distributed amplification with ultralong fiber lasers, Opt. Express, vol. 18, pp. 23569-23575, 2010.

[11] J. Nuño, M. Alcon-Camas, and J.D. Ania-Castañón: RIN transfer in random distributed feedback fiber lasers, Opt. Express, vol. 20, pp. 27376-27381, 2012.

[12] M. Tan, P. Rosa, M. A. Iqbal, I. Phillips, J. Nuño, J. D. Ania-Castanon, and P. Harper: RIN mitigation in second-order pumped Raman fibre laser based amplification, in Proc. ACP 2015, Hong Kong, China, Nov. 2015, paper AM2E.6.

[13] G. Rizzelli, M. A. Iqbal, F. Gallazzi, P. Rosa, M. Tan, J. D. Ania-Castañón, L. Krzczanowicz, P. Corredera, I. Phillips, W. Forysiak, and P. Harper: Impact of input FBG reflectivity and forward pump power on RIN transfer in ultralong Raman laser amplifiers, Opt. Express, vol. 24, pp. 29170-29175, 2016. 